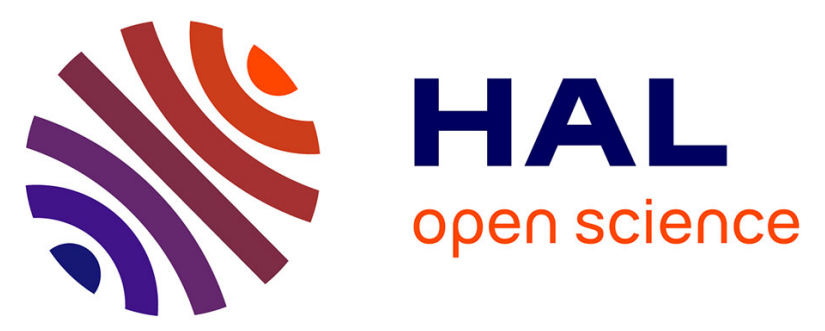

\title{
Needle, la navigation web contributive comme modalité d'accès éthique aux documents numériques
}

\author{
Julien Falgas
}

\section{To cite this version:}

Julien Falgas. Needle, la navigation web contributive comme modalité d'accès éthique aux documents numériques. 6e conférence Document numérique \& Société. Information-communication: le recours à l'éthique en contexte numérique, Dicen-IDF (Conservatoire national des arts et métiers, Université Paris-Est Marne-la-Vallée, Université Paris-Ouest Nanterre La Défense); Groupe de recherche sur les enjeux de la communication (Gresec, Université Grenoble-Alpes), Sep 2018, Echirolles, France. pp.115-126. hal-01872127

\section{HAL Id: hal-01872127 \\ https://hal.univ-lorraine.fr/hal-01872127}

Submitted on 19 Dec 2018

HAL is a multi-disciplinary open access archive for the deposit and dissemination of scientific research documents, whether they are published or not. The documents may come from teaching and research institutions in France or abroad, or from public or private research centers.
L'archive ouverte pluridisciplinaire $\mathbf{H A L}$, est destinée au dépôt et à la diffusion de documents scientifiques de niveau recherche, publiés ou non, émanant des établissements d'enseignement et de recherche français ou étrangers, des laboratoires publics ou privés. 
needle, la navigation web contributive comme modalité d'accès éthique aux documents numériques

\section{needle, la navigation web contributive comme modalité d'accès éthique aux documents numériques}

Julien FALGAS, CREM, Université de Lorraine

julien.falgas@gmail.com

\section{Résumé :}

Imaginé dans le cadre d'une recherche-action, le projet needle interpelle la responsabilité du monde universitaire. Il fait l'hypothèse de la primauté de l'intelligence humaine et collective et poursuit une visée utopique assumée. Chacune de ces dimensions éthiques implique des choix fonctionnels, techniques et stratégiques. C'est ainsi que, sous la forme d'une extension de navigateur, needle se distingue non seulement des formes les plus répandues de l'infomédiation que sont le moteur de recherche ou le réseau social, mais également des outils de veille ou de gestion de signets. Tout en s'articulant avec les outils et les pratiques existants, needle échappe donc aux définitions communes et invite, par l'exemple, à diversifier l'offre fonctionnelle en prenant acte des limites des solutions existantes.

Mots-clés : infomédiation, responsabilité, intelligence collective, utopie, contribution 
needle, la navigation web contributive comme modalité d'accès éthique aux documents numériques

\section{Des constats convergents entre chercheurs et praticiens}

La tendance des GAFAM (Google, Amazon, Facebook, Apple, Microsoft) à dominer nos pratiques d'accès aux documents numériques en ligne semble aujourd'hui établie pour tout un courant critique en sciences de l'information et de la communication (Miège, 2017; Smyrnaios, 2017 ; Ertzscheid, 2017). Profitant de l'impensé qui consiste à marginaliser les questionnements autour des enjeux de sociétés que soulève le numérique (Robert, 2016), ces infomédiaires (Rebillard et Smyrnaios, 2010) masquent leurs orientation éditoriales (Simonnot, 2016) derrière une illusion de neutralité technologique : dans le choix de ce qui est publié ou non, leurs algorithmes opaques (Cardon, 2015) ont pris le pas sur les chartes éditoriales publiques des médias. Sur le plan économique, tous secteurs éditoriaux confondus, on constate que cela se traduit par une paupérisation et une précarisation des acteurs les plus fragiles que sont les auteurs et journalistes (Charon, 2015 ; Martel, 2015 ; Paris et al. 2016). En outre, l'individu porteur d'une information, d'une idée, d'une création, peut de moins en moins les offrir à la connaissance de la société hors de ces écosystèmes, quand bien même s'inscriraitil dans un régime exclusivement vocationnel (Heinich, 2008). Le document numérique se voit privé de sa fonction de medium qui permet de le considérer «comme un phénomène social, un élément tangible d'une communication entre des personnes humaines » (Pédauque, 2003 : 18).

Une telle situation pose la question de la ré-invention du service Internet le plus répandu, le World Wide Web (Stiegler, 2017) face au constat qu'un vice-président de la foundation Mozilla pose en ces termes : " the tragedy of the web is that it's hard to find the answers to the questions you haven't imagined, and expanding your imagination has only gotten harder now that both search and social networks strive to give you a heavily filtered version of the web $\gg 1$ (Guyen, 2017). A l'occasion des 29 ans de son invention, Tim Berners-Lee, a estimé que le Web était menacé et que sa défense nécessitait de dépasser deux mythes : "le mythe que la publicité est le seul modèle commercial possible pour les entreprises en ligne, et le mythe selon lequel il est trop tard pour changer le mode de fonctionnement des plates-formes » (Berners-Lee, 2018). Pour le président du World Wide Web Consortium (W3C) c'est bien d'un manque d'imagination dont nous serions victimes. Un manque d'imagination qu'incrimine également le sociologue Dominique Cardon en considérant qu' «il appartient aux chercheurs, aux communautés, aux usagers, aux pouvoirs publics aussi, d'encourager les initiatives qui préservent cette dynamique réflexive, polyphonique et peu contrôlable que les pionniers du web nous ont confiée » (Cardon, 2017).

L'issue de notre recherche doctorale (Falgas, 2014) nous a conduit à établir ces constats. Les formes narratives innovantes que nous étudiions ne parvenaient pas à s'installer durablement. Or, ce n'était

\footnotetext{
${ }^{1}$ «La tragédie du web c'est qu'il est difficile de trouver des réponses à des questions que l'on n'a pas imaginées, tandis que faire preuve d'imagination est devenu de plus en plus difficile à mesure que moteurs de recherche et réseaux sociaux nous offrent une version lourdement filtrée du web. » (traduction personnelle)
} 
needle, la navigation web contributive comme modalité d'accès éthique aux documents numériques

pas faute d'être l'œuvre d'auteurs animés par d'authentiques ambitions narratives bien plus que par le souci de l'innovation formelle. Ce n'était pas faute non plus de trouver des publics engagés et animés par des noyaux de fans. C'était faute d'un écosystème numérique suffisamment accueillant pour la création. Progressivement, c'est la source même de l'imagination qui se tarit : nous sommes face à une crise de l'inspiration (Falgas, 2018).

\section{Démonstration du service de navigation web contributive needle}

En réponse à ces constats, et dans une démarche de recherche-action engagée, nous avons imaginé le service en ligne needle dont l'expérimentation à l'échelle de l'Université de Lorraine a débuté en 2018. Afin de rendre compte de la démonstration de ce service, nous en reproduisons le descriptif fonctionnel.

Fig. 1 - Descriptiffonctionnel de la navigation contributive proposée par needle

needle se présente comme une extension de navigateur. Elle sera disponible à l'adresse http://needle.univ-lorraine.fr à l'issue de la phase d'expérimentation actuellement en cours.

Suite à l'installation de l'extension, l'utilisateur de needle dispose d'un bouton en forme d'aiguille sur lequel il peut cliquer - au cours de sa navigation - lorsqu'il souhaite indexer une page web le long de son « fil » (Fig. 2). Tout en enrichissant son propre « fil », cette indexation ouvre à l'utilisateur l'accès immédiat aux « fils » des autres utilisateurs qui ont choisi d'indexer la même page que lui auparavant (Fig. 3). L'utilisateur peut ainsi découvrir de nouvelles ressources qui ont pour double caractéristique d'avoir été successivement indexées par quelqu'un d'autre, et de l'avoir été pour des raisons similaires : à savoir pour s'en servir comme sources de sérendipité. Ainsi l'utilisateur est-il invité à indexer des pages qui l'inspirent, dans la mesure où il souhaite qu'elles le conduisent à de nouvelles sources d'inspiration.

Outre l'accès immédiat au réseau offert par l'indexation d'une page web, l'utilisateur peut à tout moment y accéder depuis chacune des pages qu'il a indexées par le passé. Dans l'interface de gestion de son « fil », il peut en effet visualiser rapidement si de nouveaux « fils » ont croisé le sien au cours d'une période de son choix (par exemple au cours de la semaine qui vient de s'écouler). Enfin, en rejoignant des groupes, l'utilisateur est en mesure de partager son cheminement avec un collectif identifié (par exemple les membres d'une unité de recherche ou d'un établissement) mais aussi d'en distinguer les autres membres par rapport à l'activité de l'ensemble des autres utilisateurs (par exemple pour s'assurer de ne pas suivre que les fils de ses plus proches connaissances ou collaborateurs). Enfin, l'utilisateur est invité à fournir une adresse électronique pour autoriser les autres à entrer en contact avec lui si son fil leur inspire de possibles synergies, collaborations ou échanges enrichissants.

Comme on le voit, needle repose sur la contribution collective à un index. Cet index référence un réseau de pages web connectées entre elles par les utilisateurs de needle et non par des liens hypertextes saisis par leurs concepteurs. Chaque page référencée par un même utilisateur est reliée à la précédente et à la suivante le long de son «fil ». Les nœuds du réseau correspondent aux pages que plusieurs utilisateurs différents ont référencées et sur lesquels se croisent leurs «fils». Ainsi, les suggestions offertes par needle au cours de la navigation de ses 
needle, la navigation web contributive comme modalité d'accès éthique aux documents numériques

utilisateurs reposent sur une logique de partage et de co-construction. C'est ce que nous avons nommé la navigation web contributive.

Fig. 2 -D'un clic sur le bouton, l'utilisateur dispose d'un panneau depuis lequel indexer lui-même la page courante le long de son «fil».

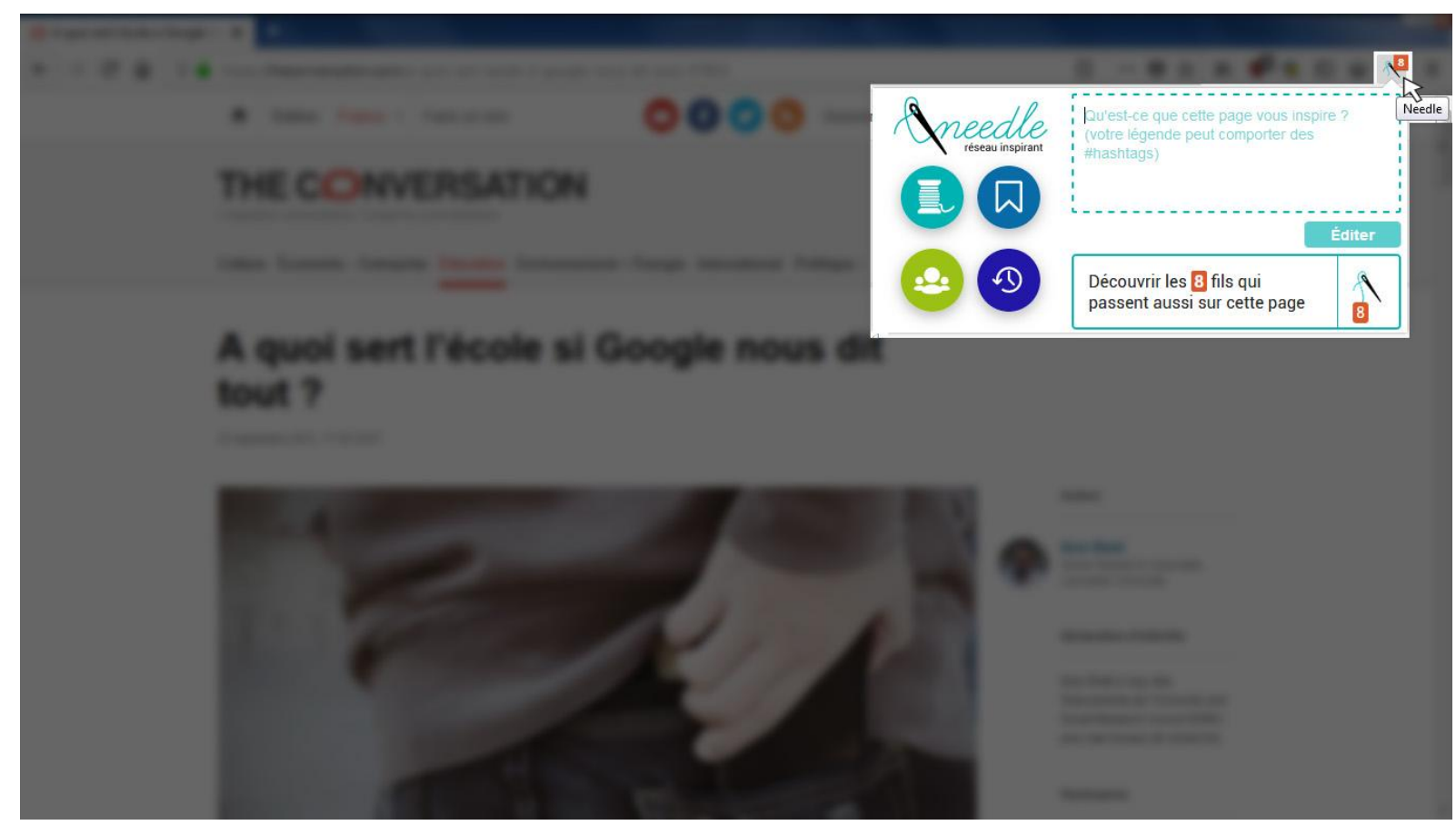

Fig. 3 - Après avoir indexé une page courante le long de son " fil », l'utilisateur accède immédiatement en retour aux « fils » des autres utilisateurs ayant indexé cette même page.

\begin{tabular}{|c|c|c|}
\hline A quoi sert r'école si Google no $x \quad$ Découvrir & $\times+$ & 미잉 \\
\hline 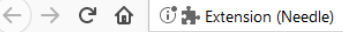 & moz-extension://5a662636-6969-45ad-895e-b22ba02e631e/discover.html?link_id = 3052148cle=10148sort_by & ... • \\
\hline Aneedle & & $\stackrel{1}{2 \downarrow} \subset$ \\
\hline & Vous croisez 8 fils sur la page & \\
\hline & A quoi sert l'école si Google nous dit tout? & \\
\hline
\end{tabular}

Fil sans titre

+ Mémoriser $\times$ Masquer ce fil

Q $\mid \square$ croisements $\mid$

\begin{tabular}{|c|c|c|c|c|}
\hline $\begin{array}{l}\text { H. Le } \\
<\end{array}$ & $\begin{array}{l}03 \text { Avril } 16 \\
\text { updates \& insights | Innovations in } \\
\text { Scholarly Communication }\end{array}$ & $\begin{array}{l}03 \text { Avril } 16 \\
\text { A quoi sert l'école si Google nous } \\
\text { dit tout? }\end{array}$ & $\begin{array}{l}04 \text { Avril } 16 \\
\text { La validation centralisée dans HAL } \\
\text { nuit-elle à son efficacité ? - } \\
\text { Assessment Librarian : un blog } \\
\text { sur les enquêtes et l'évaluation } \\
\text { des servic }\end{array}$ & $\begin{array}{l}04 \text { Avril } 16 \\
\text { Le mod } \\
\text { One du }\end{array}$ \\
\hline icanal-u.tv & \& 101 innovations.wordpress.com & \& theconversation.com & \& assessmentlibrarian.fr & \\
\hline \multicolumn{4}{|c|}{$102 / 4606$} & 02 Juil. 17 \\
\hline
\end{tabular}


needle, la navigation web contributive comme modalité d'accès éthique aux documents numériques

Les outils et les méthodes de veille créative sont variés (Goria, 2017), cependant aucun n'est de nature à démocratiser une modalité d'accès aux documents à visée créative depuis que la navigation hypertextuelle a cédé le pas à l'usage d'un moteur de recherche et à la consultation des échanges et partages de liens sur les réseaux sociaux. Depuis que les liens hypertextes ont acquis une valeur marchande au regard des critères de référencement de Google, ils ont progressivement perdu leur pertinence pour l'internaute. Parallèlement, les réseaux sociaux ont permis à tout-un-chacun de partager des liens sans saisir la moindre ligne de code et pour une gratification sociale immédiate qui occulte la portée éphémère de ces échanges. Avec le développement de ces usages, on ne surfe plus sur le Web, on y accède par un entonnoir (Simonnot et Gallezot, 2009) qu'il s'agisse des résultats fournis par le moteur de recherche ou des liens partagés sur les murs des réseaux sociaux. Les deux modes dominants de mise en visibilité des documents sur le Web nous enferment dans leurs conceptions respectives de la réputation : l'un par le lien, l'autre par le like (Cardon, 2013). Nous pourrions imaginer d'autres approches dont la pluralité contribuerait à un environnement numérique plus résilient face aux stratégies de détournement et de désinformation qui se concentrent actuellement sur une poignée d'infomédiaires.

Prenant acte de cette situation, nous portons le projet needle dans le cadre d'une recherche-action qui répond à trois dimensions éthiques que nous proposons de développer et d'expliciter ici : responsabilité et réflexivité du monde universitaire, primauté de l'intelligence humaine et collective et, enfin, la visée utopique du projet. Nous exposerons les implications de chacune quant aux choix fonctionnels, techniques et stratégiques qui sous-tendent le projet needle.

\section{Prendre en compte notre responsabilité et celle de nos organisations académiques}

Dans une perspective de développement durable au sens large et selon l'imposition d'un principe écologique (Guattari, 1989 et 1992 ; Beacker, 2007 ; Hörl, 2012), la notion d'empreinte s'est imposée à nous pour traduire l'exigence de réflexivité (Durampart, 2016) faite à nos organisations universitaires vis-à-vis des acteurs évoluant dans l'environnement numérique. Avec l'empreinte, il ne s'agit pas de rendre possible une lecture par les uns de ce que font les autres, mais d'accéder à une lecture par soi-même de ce que l'on a fait. Ainsi, l'empreinte englobe-t-elle le triptyque indice inscription - trace (Jeanneret, 2010) dans une objectivation réflexive (Appel et Falgas, 2019).

Comme le montre l'exemple de Facebook, dont la première version en 2004 s'adressait aux étudiants d'Harvard, l'empreinte des universités peut aller jusqu'à incuber des innovations de rupture à l'impact mondial. Le monde universitaire peine à reconnaître cette responsabilité. Nul n'ignore aujourd'hui l'adage selon lequel «si c'est gratuit, c'est que c'est vous le produit». Pourtant, on a pu voir cette année des établissements - dont le nôtre - accueillir des formations délivrées gracieusement par des ambassadeurs de Google recrutés parmi leurs étudiants (Stromboni, 2018). En 2016 déjà, le conseil d'administration de 1'université Paris-3 Sorbonne-Nouvelle avait maintenu sa décision d'adopter les 
needle, la navigation web contributive comme modalité d'accès éthique aux documents numériques

services d'informatique dans les nuages de Google en dépits des mises en garde de ses propres chercheurs en Sciences de l'information et de la communication (Rebillard et Rochelandet, 2016), au motif que l'offre du géant de Mountain View était gratuite. Il a fallu la publication d'une note ministérielle destinée aux collectivités territoriales pour interrompre la mise en œuvre du projet (Ministère de la culture, 2016).

Le projet needle contribue à interpeller le monde universitaire quant à sa responsabilité vis-à-vis de l'environnement numérique. Cela se traduit par le recours au financement de valorisation nonéconomique de la recherche. Ce mode de financement - d'un montant limité à quelques milliers d'euros et ne pouvant être reconduit au-delà de la deuxième année - a préservé le projet des injonctions à la lucrativité. De telles injonctions auraient impliqué des choix de conception destinés à maintenir captive l'attention des utilisateurs dans la perspective d'un service gratuit, ou bien nous auraient obligé à restreindre l'accès au service à une clientèle solvable. Nous pouvons ainsi nous attacher à concevoir un dispositif utile au monde universitaire, à ses partenaires et aux citoyens. Parmi ces besoins figure, pour les chercheurs de nos disciplines, celui de recueillir des données d'usages quant aux pratiques informationnelles des internautes. Dans la mesure où les principaux infomédiaires ne partagent leurs données qu'en fonction du bénéfice qu'ils espèrent en tirer, il nous est apparu nécessaire de mettre en œuvre une offre d'infomédiation alternative à même de réunir des données pertinentes pour nos recherches. La poursuite du projet lui-même est source de données précieuses quant aux négociations à l'œuvre autour de la «mise en boîte noire » de needle (Akrich, 1992; Callon, 2001).

\section{Faire primer l'intelligence humaine sur l'intelligence artificielle des algorithmes}

Au-delà de la portée heuristique du service, needle est avant tout un outil destiné à favoriser l'inspiration de chacun. Nous avons en effet identifié l'intérêt de needle pour répondre à des besoins tels que : compléter des recherches ou une veille par des ressources inattendues, explorer des pistes de réflexion et d'innovation à la périphérie de son champ d'expertise, ou encore initier des rencontres voire des collaborations en dehors de ses réseaux habituels.

Pour y parvenir, nous misons sur une deuxième dimension éthique de notre proposition, qui concerne la place de l'intelligence humaine dans le dispositif de recommandation de documents, par opposition à l'intelligence artificielle des algorithmes. Au regard des premières réactions recueillies suite à des démonstrations de needle devant des utilisateurs pressentis (chercheurs, entrepreneurs, acteurs du secteur de l'innovation ou des collectivités territoriales), il semble que needle constitue un outil à même de favoriser le décloisonnement, l'identification de synergies et de renforcer les collaborations. Il répond ainsi à la définition de l'intelligence collective avancée par Samuel Szoniecky et Nasreddine Bouhaï (2017), à savoir « la mise en commun de la réflexivité afin de finaliser une action qui ne pourrait pas l'être par une seule personne » (Ibid., p. 14). Samuel Szoniecky et Nasreddine Bouhaï 
needle, la navigation web contributive comme modalité d'accès éthique aux documents numériques

illustrent leur propos par l'exemple du Wiki. Si le Wiki rend possible l'exercice de l'intelligence collective autour de la composition de contenus tout en permettant leur mise en relation hypertextuelle, needle se concentre sur l'élaboration d'un réseau hypertextuel entre des contenus dont les modalités de composition et de publication en ligne restent libres. Soulignons enfin que l'interface de needle favorise la contribution collective à un index indépendant qui pourrait servir de base au développement de nouvelles solutions de recherche en ligne parmi un corpus documentaire coconstruit entre ses propres utilisateurs (Lewandowski, 2002).

D'ores-et-déjà, l'expérience d'accès aux contenus que référence needle se distingue de celle qu'offrent les plateformes traditionnelles de l'infomédiation telles que les moteurs de recherche ou les réseaux sociaux. Un moteur de recherche s'appuie traditionnellement sur la formulation d'une requête à laquelle l'algorithme cherche à répondre en puisant dans un index préalablement constitué. needle ne nécessite pas de formuler une requête : il suffit de cliquer sur un bouton pour être mis en relation avec des contenus connexes auxquels nous n'aurions pas toujours su accéder par une requête, ni même eu l'idée de le faire. Sur un réseau social, nous établissons des liens entre notre profil et ceux d'autres utilisateurs de notre connaissance. Ces liens peuvent être plus ou moins solides selon qu'ils découlent d'une relation établie (amicale, familiale, professionnelle, ...) mais ils reposent toujours sur notre réseau relationnel existant, fut-il à sens unique (comme lorsqu'on se connecte au profil d'une célébrité). En outre, les informations que nous adresse le réseau social sont inscrites dans l'immédiateté : elles visent à nous maintenir informé de l'activité et de l'actualité de notre réseau. Contrairement à un réseau social, needle nous expose à des contenus indexés par des inconnus, parfois plusieurs jours, mois ou années avant ou après nous. Ajoutons que needle ne met pas en avant un profil, mais seulement un fil : l'identité de l'utilisateur est secondaire, le service est d'ailleurs conçu à l'origine pour un usage anonyme. Il n'y a donc pas d'enjeu à afficher le fruit de sa veille dans une stratégie de gestion de son identité numérique : le seul intérêt à indexer une référence réside dans le désir d'en faire un point de rencontre potentiel avec d'autres références aussi inspirantes.

Les utilisateurs plus avancés pourront relever une proximité de needle avec les outils de veille ou de gestion de signets. Les différents outils de veille visent cependant à nous maintenir informés de l'actualité, mais aussi à le faire au regard de sphères d'intérêts et de thématiques prédéfinies. Ces outils reposent sur une sélection préalable de sources au regard de critères tels que la fiabilité, la pertinence ou l'expertise dans tel ou tel domaine. Contrairement à un outil de veille, needle ne présume pas de nos sphères d'intérêt et des thématiques susceptibles de déterminer quels contenus sont susceptibles de nous inspirer. Enfin, des outils de signets - qu'ils soient sociaux ou non permettent d'archiver et d'organiser le fruit de nos recherches, des partages dont nous avons bénéficié sur les réseaux sociaux ou encore de notre veille. Cet archivage vise essentiellement à conserver une trace à laquelle se référer dans le futur si l'on ressent le besoin de revenir à une information ou une donnée dont on a conservé le souvenir. Contrairement à un outil de signets, et bien qu'il le permette 
needle, la navigation web contributive comme modalité d'accès éthique aux documents numériques

dans une certaine mesure, needle ne vise pas tant à organiser un archivage destiné à être exploré ultérieurement, mais réactive les ressources indexées par le passé au regard de l'intérêt qu'elles suscitent pour d'autres - y compris dans un temps long. Ajoutons que needle ne repose pas sur la folksonomie et n'attend pas de ses utilisateurs qu'ils associent des mots-clés ou des étiquettes aux références qu'ils indexent: nous faisons l'hypothèse qu'indexer une référence apporte un sens suffisant à la mise en œuvre de la navigation web contributive, puisque la motivation de cette action est similaire pour chaque utilisateur. La reconnaissance de mots-dièses au sein des légendes associées aux références que l'on indexe est toutefois envisagée à l'attention des utilisateurs désireux d'organiser plus finement leur fil sans devoir recourir parallèlement à un outil de signets plus évolué.

Tout en s'articulant avec les outils et les pratiques existants, needle échappe donc aux définitions communes. De ce fait, le service offre un moyen de réaliser l'exploration curieuse à laquelle nous incite Nicolas Auray (2016) par opposition à l'enfermement algorithmique auquel concourent les plateformes numériques dominantes. Pour y parvenir, needle mise sur le partage et la co-construction des connaissances bien plus que sur la puissance de calcul informatique.

A mesure que needle se développera, nous disposerons de données suffisantes pour les soumettre à des traitements informatiques. L'enjeu consistera alors à préserver la primauté du choix humain conscient sur le traitement par la machine destinée à fournir une recommandation calculée. Pour l'heure, à partir d'une référence donnée le long du fil de l'utilisateur, needle recommande le premier fil à avoir jamais indexé cette même référence. Il est possible de masquer cette recommandation pour voir la suivante. Il est également possible d'inverser le tri pour prioriser les derniers fils à avoir indexé la référence en question. A l'avenir, nous devrons pouvoir choisir de discriminer ou de prioriser les fils qui nous seront recommandés en fonction de critères tels que leur pertinence au regard de l'intérêt que leur portent d'autres utilisateurs, la diversité des sources indexées, ou encore le fait que leurs auteurs appartiennent aux mêmes groupes que nous ou non. L'identification des critères pertinents et les modalités de contrôle offertes aux utilisateurs sur leur mise en œuvre constituent un horizon de recherche à part entière qui reste à explorer.

\section{Un projet utopique fondé sur l'engagement du monde universitaire}

Sur le plan technologique, needle est destiné à une diffusion open source au sein d'une architecture distribuée (c'est-à-dire non dépendante d'un acteur central et dominant). Le fonctionnement du service ne nécessite la collecte d'aucune donnée personnelle. Ces caractéristiques attestent de la troisième dimension éthique qui préside à cette innovation : la dimension utopique du projet.

Pour Patrice Flichy (2001), l'utopie qui n'affronte pas la réalité technique relève de la fantasmagorie. S'appuyant sur Paul Ricoeur (1997) il définit l'utopie comme « une alternative au pouvoir en place » (Ibid., p. 14). C'est par la déclinaison en projet d'une proposition de rupture, par la transformation de l'expérience réussie en mythe, que l'utopie-fantasmagorie de l'inventeur peut devenir une idéologie. 
needle, la navigation web contributive comme modalité d'accès éthique aux documents numériques

Si les interfaces hommes-machine matérialisent l'utopie d'un esperanto visuel (Manovitch, 2001), encore faut-il que les règles qu'elles fixent à ce langage universel soient porteuses d'utopie. Dans cette perspective, needle ne vise ni à occulter (idéologie-masque) ni à évincer (idéologie-légitimante) les formes actuelles d'accès aux documents numériques en ligne mais à mobiliser (idéologiemobilisation) autour d'une forme alternative plus proche de l'utopie fondatrice du World Wide Web (Faucilhon, 2010) que partageaient ceux qui l'en ont pourtant éloigné : «we believe the issue of advertising causes enough mixed incentives that it is crucial to have a competitive search engine that is transparent and in the academic realm ${ }^{2} \gg$ (Brin \& Page, 2000, p. 3832), fut-elle limitée au fait d'apporter des changements à portée immédiate (Proulx et al., 2005).

Au-delà du développement de moteurs de recherche ou de réseaux sociaux open source et/ou distribués à même de concurrencer les acteurs dominants, needle invite à diversifier l'offre fonctionnelle en prenant acte des limites des solutions existantes. Ce faisant, il s'agit d'interpeller la responsabilité du monde universitaire quant à l'avenir du web au travers d'une réalisation concrète. La part utopique d'un tel projet semble constituer sa limite la plus évidente. De prochains travaux interrogeront les tenants et les aboutissants, au regard des origines de l'utopie numérique, d'une telle tentative de matérialiser une utopie. Il conviendra par ailleurs à l'avenir d'expliciter le positionnement de cette nouvelle voie au sein des sciences de l'information et de la communication, par rapport à la tradition critique qui a contribué à faire émerger et à qualifier finement les enjeux auxquels needle se confronte.

\section{Bibliographie}

- AKRICH (M.), La description des objets techniques dans AKRICH (M.), CALLON (M.) et LATOUR (B.), Sociologie de la traduction: textes fondateurs, Paris, Mines Paris, Les Presses, 1992 et 2006.

- APPEL (V.) et FALGAS (J.), «Responsabilité sociétale des universités et environnement numérique : la notion d'empreinte, un enjeu de réflexivité », Communication, vol. 35/2, 2019, A paraître.

- AURAY (N.), L'Alerte Ou L'enquête. Une Sociologie Pragmatique Du Numérique, Paris: Presses des Mines, 2016.

- BEACKER (D.), Studien zur nächsten Gesellschaft, Francfort/Main, 2007.

- BERNERS-LEE (T.) (2018, 12 mars) «The web is under threat. Join us and fight for it» Sur le site de la World Wide Web Foundation. Consulté le 24 avril 2018.

\footnotetext{
2 Traduction personnelle : «nous croyons que le modèle publicitaire est à l'origine d'un nombre suffisant d'incitations biaisées pour qu'il soit crucial de disposer d'un moteur de recherche compétitif à la fois transparent et relevant du monde académique ».
} 
needle, la navigation web contributive comme modalité d'accès éthique aux documents numériques

https://webfoundation.org/2018/03/web-birthday-29/

- BRIN (S.) et PAGE (L.), « Reprint of: The anatomy of a large-scale hypertextual web search engine », Computer networks 56.18 (2012), 2000/2012, pp. 3825-3833.

- CALlON (M.), Sociologie de l'acteur réseau, traduit de l'anglais par CALLON (G.) dans AKRICH (M.), CALLON (M.) et LATOUR (B.), Sociologie de la traduction: textes fondateurs, Paris, Mines Paris, Les Presses, 2001 et 2006.

- CARDON (D.), «Du lien au like sur Internet: Deux mesures de la réputation», Communications 93, vol. 93, no. 2, 2013, pp. 173-186.

- CARDON (D.), A Quoi Rêvent Les Algorithmes: Nos Vies À L'heure Des Big Data, Paris: La République des idées : Seuil, 2015.

- CARDON (D.) Le web que nous voulons en 8 propositions dans STIEGLER (B.) (dir.), La toile que nous voulons: le web néguentropique, Paris, FYP, 2017.

- CHARON (J.-M.) (2015, 2 juin), Presse et Numérique - L'invention D'un Nouvel Écosystème. Rapport remis à Madame la Ministre de la culture et de la communication. Consulté le 24 avril 2018.

http://www.culturecommunication.gouv.fr/Ressources/Rapports/Rapport-Charon-Presse-etnumerique-L-invention-d-un-nouvel-ecosysteme

- DURAMPART (M.), Le passage d'un enjeu cognitif à un "hors-jeu" stratégique pour des organisations, dans BONFILS (P.), DUMAS (P.) et MASSOU (L.) (dirs), Dispositifs, jeux, enjeux, hors-jeux, Nancy, PUN - Éditions universitaires de Lorraine, 2016.

- ERTZSCHEID (O.), L'appétit des géants, Pouvoir des algorithmes, ambitions des plateformes, Paris, C\&F Editions, 2017.

- FALGAS (J.), Raconter à l'ère numérique : auteurs et lecteurs héritiers de la bande dessinée face aux nouveaux dispositifs de publication, Thèse de doctorat en Sciences de l'information et de la communication, Université de Lorraine, 2014.

- FALGAS (J.) «needle, une innovation issue des sciences de l'information et de la communication face à la crise de l'inspiration » dans Actes du XXIe congrès de la SFSIC, mai 2018, Paris, 2018.

- FAUCILHON (J.), Rêveurs, Marchands et Pirates Que Reste-T-Il Du Rêve de l'Internet?, Le Pré Saint-Gervais: Ed. le passager clandestin, 2010.

- FLICHY (P.), L'imaginaire d'Internet, Sciences et Société. Paris: Découverte, 2001.

- GORIA (S.), Méthodes et outils de veille créative, iSTE editions, Londres, 2017.

- GUATTARI (F.), Les Trois Écologies, Paris, éditions Galilée, 1989 et 2008.

- GUATTARI (F.), Le nouveau paradigme esthétique, dans GUATTARI (F.), Chaosmose, Paris, Editions Galilée, coll. L'Espace critique, 1992. 
needle, la navigation web contributive comme modalité d'accès éthique aux documents numériques

- GUYEN (N.), (2017, 19 janvier), «Your phone is not a television » sur le blog du projet Firefox Context Graph. Consulté le 24 avril 2018.

https://medium.com/firefox-context-graph/your-phone-is-not-a-television-2aaae67c645

- HEINICH (N.), Régime vocationnel et pluriactivité chez les écrivains : une perspective compréhensive et ses incompréhensions, dans Socio-logos, 3, 2008.

- HÖRL (E.), Le nouveau paradigme écologique: Pour une écologie générale des médias et des techniques, traduit de l'allemand par PLAS (G.), dans Multitudes, 2012/4 (n 51), 2012, p. 74 85 .

- JEANNERET (Y.), Les harmoniques du Web: espaces d'inscription et mémoire des pratiques, dans PIGNIER (N.) et LAVIGNE (M.) (dirs.), Mémoires et internet, Paris, Harmattan, 2010.

- LEWANDOWSKI (D.) Why We Need an Independent Index of the Web, dans Networks vol. $39, n^{\circ} 3,2002$, p. 289-302.

- MANOVITCH (L.), Le langage des nouveaux médias, traduit de l'anglais par CREVIER (R.), Dijon: les Presses du réel, 2001.

- MARTEL (F.) (2015, 11 septembre). L'écrivain 'social' : La Condition de L'écrivain À L'âge Numérique. Rapport au CNL. Consulté le 24 avril 2018.

http://centrenationaldulivre.fr/fichier/p_ressource/7429/ressource_fichier_fr_condition.a.crivai n.monde.numa.rique.rapport.2015.11.09.ok.pdf

- Ministère de la culture. (2016, 5 avril). Note d'information relative à l'informatique en nuage (coud computing). Consulté le 24 avril 2018.

http://securibase.com/securibase/public/fiche/19224/21193

- MIEGE (B.), Les industries culturelles et créatives face à l'ordre de l'information et de la communication. Nouvelle édition entièrement refondue et augmentée, Collection communication en plus, Fontaine: Presses universitaires de Grenoble, 2017.

- PÉDAUQUE (R. T.) (2003, 8 juillet), «Document: forme, signe et médium, les reformulations du numérique ». Consulté le 24 avril 2018.

https://archivesic.ccsd.cnrs.fr/sic_00000511

- PARIS (T.), DELOCHE (P.) et SIMON (M.) (2016, 29 janvier). Etats Généraux de La Bande Dessinée, Enquête Auteurs 2016, Résultats Statistiques. Consulté le 24 avril 2018. http://www.etatsgenerauxbd.org/2016/01/29/lenquete-auteurs-les-resultats-statistiques/

- PROULX (S.), MASSIT-FOLLEA (F.), CONEIN (B.), Internet, une utopie limitée: nouvelles régulations, nouvelles solidarités, Québec: Presses de l’Université Laval, 2005.

- REBILlaRD (F.) et SMYRNAIOS (N.), Les infomédiaires, au cœur de la filière de l'information en ligne: Les cas de google, wikio et paperblog, dans Réseaux, vol. 160-161, $\mathrm{n}^{\circ}$ 2, 2010, pp. 163-194. 
needle, la navigation web contributive comme modalité d'accès éthique aux documents numériques

- REBILLARD (F.) et ROCHELANDET (F.) (2016, 3 juin), « Lettre ouverte aux membres du Conseil d'Administration de l'Université Sorbonne Nouvelle Paris 3 », Google à Paris 3 ? Non, merci, Consulté le 4 septembre 2018. https://googlep3.wordpress.com/2016/06/03/lettreouverte-aux-membres-du-conseil-dadministration-de-luniversite-sorbonne-nouvelle-paris-3/

- RICOEUR (P.), L'idéologie et l'utopie, Paris, éditions du Seuil, 1997.

- ROBERT (P.), L'impensé numérique - Tome 1, Des années 1980 aux réseaux sociaux, Paris, Éditions des Archives contemporaines, 2016.

- SIMONNOT (B.) et GALLEZOT (G.) (dirs.), L'entonnoir: Google sous la loupe des sciences de l'information et de la communication, Caen: C\&F, 2009.

- SIMONNOT (B.), Place des moteurs de recherche dans l'éditorialisation du web, dans Communication \& langages, Nec Plus, $\mathrm{n}^{\circ}$ 188, 2016, pp.45-59.

- SMYRNAiOS (N.), Les GAFAM contre l'internet, Paris, Ina éditions, 2017.

- STIEGLER (B.) (dir.), La toile que nous voulons: le web néguentropique, Paris, FYP, 2017.

- STROMBONI (C.) (2018, 5 mars), «Des formations au numérique dispensées par Google à l'université font débat», Le Monde, Campus. Consulté le 24 avril 2018. http://www.lemonde.fr/campus/article/2018/03/05/des-formations-au-numerique-dispenseespar-google-a-1-universite-font-debat_5265898_4401467.html

- SZONIECKY (S.), et BOUHAÏ (N.), Intelligence collective et archives numériques: vers des écosystèmes de connaissances, iSTE editions, Londres, 2017. 